Obadă B. , Șerban Al. O., Botnaru V., Valcu Al.

\title{
The use of MRI and GT in Imaging Occult Hip Fractures
}

\author{
Clinic of Orthopedics and Traumatology, Emergency County Hospital Constanța, Romania
}

\begin{abstract}
Diagnosis of hip fractures is particularly important due to the high dependence on the integrity of this structure for people to function in their daily lives. Left unrecognized, patients face increasing morbidity and mortality as time from the original injury lengthens. A delay of just 2 days in surgical treatment for an acute hip fracture doubles mortality. In addition, an unrecognized non-displaced fracture may displace, requiring surgery of much higher risk. This may be part of the reason that the most frequent lawsuit against Emergency Physicians is for missed orthopedic injury. We reviewed the use of MRI and CT for occult hip fractures (OHF) detection at a major urban trauma unit. Our study is a retrospective review. Inclusion criteria: all patients presenting to the Emergency Clinical Hospital of Constanta with a suspected, posttraumatic, occult hip fracture, over a 5 years period were included. All patients had negative initial radiographs and underwent further imaging with either CT or MRI. A total of 185 cases meeting the inclusion criteria were identified. 72 occult hip
\end{abstract}

\section{Obada Bogdan}

Clinic of Orthopedics and Traumatology

Emergency County Hospital Constanța, Romania

bogdanobada@yahoo.com fractures were detected with both imaging modalities. Although MRI certainly enables greater image detail, in our experience both modalities are able to provide satisfactory fracture characterization. The choice of imaging should be determined by availability and indication. MRI provides superior imaging of soft tissue but is less sensitive for degenerative changes in presence of bone edema.

Keywords: occult hip fracture, CT, MRI, diagnosis

\section{Introduction}

Most of the elderly patients who suffered a fracture sustain hip fracture and the estimated cost of the treatment are very high. Early surgical intervention is recognized to be important in improving outcomes following hip fractures [1,2]. Observational studies have suggested the mortality rate doubles when the operation is performed after 2 days. Initial X-ray are negative or equivocal in $2-10 \%$ of patients presenting with posttraumatic hip fracture leading to diagnostic and treatment delays [3]. Much of the available literature advocates magnetic resonance imaging (MRI) for further investigation of such patients. However, is not readily available in all hospitals and 
computed tomography $(\mathrm{CT})$ may be more appropriate to obtain a rapid diagnosis $[4,5,6]$.

We reviewed the use of MRI and CT for occult hip fractures (OHF) detection at a major urban trauma unit.

\section{Material and Methods}

Our study is a retrospective review.

Inclusion criteria: all patients presenting to the Emergency Clinical Hospital of Constanta with a suspected, posttraumatic, occult hip fracture, over a 5 years period were included. All patients had negative initial radiographs and underwent further imaging with either CT and MRI.

Exclusion criteria: outpatients and scans for chronic issues.

\section{Results}

Data was abstracted from patient notes for demographics and comorbidities. Hospital records were checked to ensure no patients had re-attended within 12 months with a fracture that could have been missed by initial imaging.

The Statistical Package for the Social Science SPSS v15 (SPSS Inc., Chicago, Il, USA) was used to analyze data. Chi-square test or Fisher's exact test were used to analyze categorical variables. T-test was used for continuous variables. Multivariable logistic regression models were estimated for fall-related and bone-disease factors in OHF positive versus negative patients. The probability value was used as criterion for significance at $\mathrm{p} \leq 0.05$.

A total of 185 cases meeting the inclusion criteria were identified. 72 occult hip fractures were detected with both imaging modalities (Figure 1).

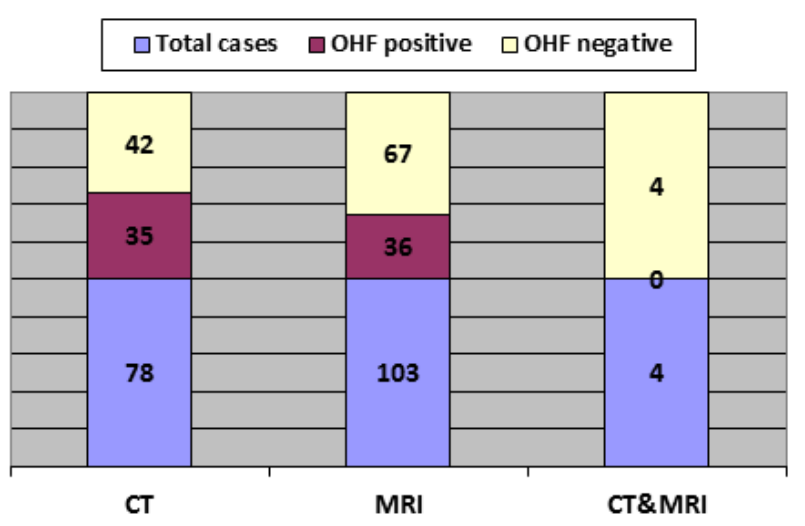

Figure 1 - Occult hip fractures identified with imaging modality

\section{Discussions}

At our center more MRI scans than CT scans were performed 103 vs 78.4 patients underwent both MRI and CT and have been excluded from final analysis. There were 118 female and 63 male patients included in this study with a mean age of $78.09 \pm$ 16.64. $85 \%$ of patients presented within 24 hours of injury or accident. Baseline characteristics of OHF positive or negative patients are compared in Table I.

Table I. Baseline characteristics of study polupation

\begin{tabular}{|l|c|c|}
\hline & $\begin{array}{c}\text { OHF negative } \\
(109)\end{array}$ & $\begin{array}{c}\text { OHF positive } \\
(72)\end{array}$ \\
\hline Sex $(\mathrm{M} / \mathrm{F})$ & $36 / 73$ & $27 / 45$ \\
\hline Mean age & $79.05 \pm 13.82$ & $79.57 \pm 13.38$ \\
\hline $\begin{array}{l}\text { Presentation } \\
<24 \mathrm{~h}\end{array}$ & $92 / 109$ & $57 / 72$ \\
\hline
\end{tabular}

The average delay to further imaging from presentation for both imaging techniques was $2.92 \pm 2.00$ days. There was a statistical difference for delay to further imaging between MRI and CT 
scans, $3.82 \pm 0.34$ and $1.74 \pm 0.19$ days, $\mathrm{p} \leq 0.05$. These numbers take into account time to request scan after initial X-rays and time to execute request. In the MRI arm all fractures were visualized on coronal $\mathrm{T} 1$ and axial STIR images [7,8].

Sub capital OHF fractures constituted the largest positive diagnosis on imaging. The fracture patterns detected are illustrated in Figure 2.
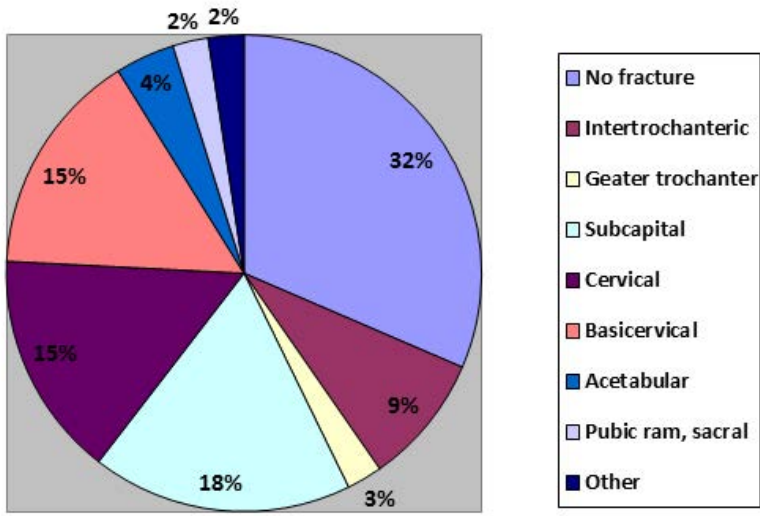

Figure 2 - Fracture patterns identified

Three of the patients undergoing both scans in our dataset were initially investigated with MRI for hip fracture based on presentation, but subsequent CT images identified severe degenerative changes. One patient undergoing both scans, underwent $\mathrm{CT}$ after MRI due to claustrophobia.

Negative MRI diagnosis are illustrated in Figure 3. $73.4 \%$ fractures detected underwent an operative procedure.

There were no further presentations to hospital with unidentified fractures within a 12 months period.

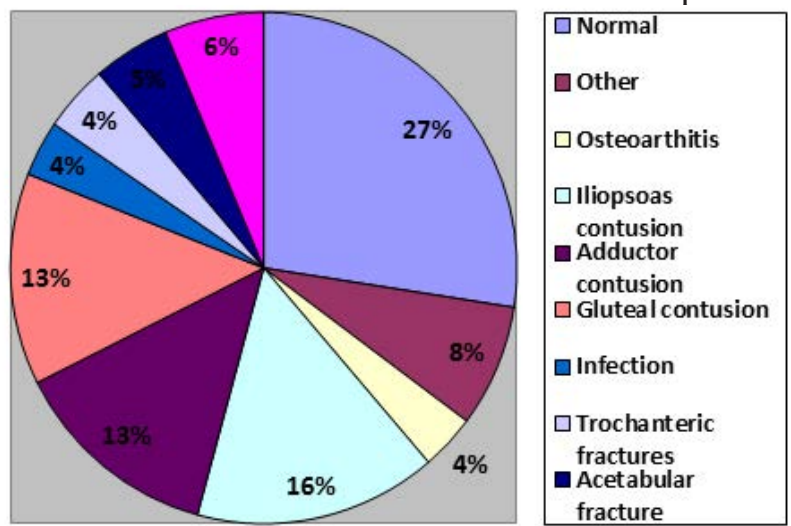

Figure 3 - Negative MRI diagnosis

\section{Conclusion}

Although MRI certainly enables greater image detail, in our experience both modalities are able to provide satisfactory fracture characterization. The choice of imaging should be determined by availability and indication. MRI provides superior imaging of soft tissue but is less sensitive for degenerative changes in presence of bone edema.

CT is a shorter examination, however, the use of fewer MRI sequences results in a shorter scanning time, previously audited at approximately 7 minutes in our department, with a total of 15 minutes room time.

There were no "missed fractures" with either CT or MRI in our study group. Accessibility to MRI should therefore not cause a delay of diagnosis and treatment beyond 24 hours.

\section{References}

1. Perron, A.D., Miller, M.D. \& Brady, W.J. (2002). Orthopedic pitfalls in the ED: radiographically occult hip fracture. Am J Emerg Med. 20, 234237

2. Pandey, R., McNally, E. \& Ali, A. et al. (1998). The role of MRI in the diagnosis of occult hip fractures. Injury. 29, 61-63

3. Lubovsky, O., Liebergall, M. \& Mattan, Y. et al. (2005). Early diagnosis of occult hip fractures MRI versus CT scan. Injury. 36, 788-792

4. Lim, K.B., Eng, A.K., Chng, S.M., Tan, A.G., Thoo, F.L. \& Low, C.O. (2002). Limited magnetic resonance imaging (MRI) and the occult hip fracture. Ann Acad Med Singapore. 31, 607-610

5. Verbeeten, K.M., Hermann, K.L. \& Hasselqvist, M. et al. (2005). The advantages of MRI in the detection of occult hip fractures. Eur Radiol. 15, 165-169 
6. Guanche, C.A., Kozin, S.H. \& Levy, A.S. et al. (1994). The use of MRI in the diagnosis of occult hip fractures in the elderly: a preliminary review. Orthopedics. 17, 327-330

7. Hossain, M., Barwick, C. \& Sinha, A.K. et al. (2007). Is magnetic resonance imaging (MRI) necessary to exclude occult hip fracture?. Injury. 38, 1204-1208
8. Quinn, S.F. \& McCarthy, J.L. (1993). Prospective evaluation of patients with suspected hip fracture and indeterminate radiographs: use of T1weighted MR images. Radiology. 187, 469-471 